\title{
Facing Neglect after Stroke: Clinical Challenges in Developing Countries Muwafak H Al-Eithan*
}

Clinical Psychology, Prince Sultan Military Medical City, Riyadh, Saudi Arabia

\begin{abstract}
Not only stroke is very prevalent, also neglect is commonly seen in acute and post-acute stages of CVA. Neglect is not a single entity neither a homogenous clinical presentation. It may cover a wide range of sensory and motor impairments. Here we raise awareness to the fact that clinically this disorder is 'neglected' as a clinical presentation partly due to its complications. Neglect is the best single predictor of recovery, and hence, the importance of dealing with it soon. Rehabilitation approaches have shown some benefits. Rehab teams need to face it, measure it and deal with it applying treatment approaches.
\end{abstract}

Keywords: Anosognosia; Stroke; Neuropsychological; Avoidant; Neglect

\section{Introduction}

Stroke is a major public health problem in the USA, and globally, ever year about 795000 people in the USA have a stroke [1]. Of the 15 million people worldwide who suffer a stroke annually, at least 5 million are permanently disabled, placing a burden on family and community [2].

Stroke has many clinical features and complications. Neglect is widely seen in clinical presentations of patients with stroke (but not exclusively), this can be between $30-70 \%$ [3].

Neglect as a neuropsychological disorder is major deficit in which the patient with right hemisphere stroke is not aware or not responding to their left side, the contralesional side $[4,5]$, without a sensory or motor deficits. Neglect also may involve sensory as well as spatial domain. The patient may show failure on intention-action aspect resulting in impaired attention, to the left side of his body often.

Clinically neglect may be presented in different subtypes: it may affect attention and perception versus intention and action. Patients with neglect, therefore, may exhibit directional hypokinesia for actions into and toward contralesional hemispace, whereas others may fail to respond to stimuli on the left of the eyes, head, or body, regardless of the required motor response [6]

On other hand, neglect may affect the contralesional body (personal neglect), contralesional near space within reaching distance (peripersonal neglect), or space beyond reaching distance (extrapersonal neglect) [6]. Nonlateralized deficits in attention may be prominent, and an important influence on its severity and persistence [7]. Neglect patients may be severely impaired in detecting targets in both hemi spaces, and may perform poorly on simple tone-counting tasks measuring non-lateralized attention. Somatosensory, visual field, and motor deficits are all more frequent after right than left hemisphere stroke, suggesting that primary sensory deficits may be augmented by neglect [7]. Anosognosia, or unawareness of deficit, is also more frequent after right than left hemisphere lesions, and may compound the disability deriving from the neglect itself $[5,7]$.

Neglect disorder is often associated with a number of other cognitive neuropsychological disorders as well as sensory and motor problems [8]. Patient may not be aware of their deficit either.

Neglect can be in a range of intensity, and not homogenous. It can be that a patient can copy a figure properly or shave beard completely or eat from the entire dish presented to him, while other patients show inability to attend to part of the face...etc.
The unawareness of deficit (anasagnosia) can complicate the clinical picture, but it may be considered as another disorder. Anasoagnosia is not a specific disorder of stroke however; it can be seen in other neurological diseases (e.g. TBI and brain tumour). Clinicians may mix them up and focus on one disorder, while ignoring the other.

Neglect has been associated with right brain damage, and it has been shown that right Temporal lobe and Parietal lobe are involved [9]. There are a number of theories that account for the deficits $[4,5]$.

Neglect is the most single predictor of outcome after stroke [5] Therefore, it is important clinically to evaluate patients after stroke and assess the possibility of the presence of neglect and its severity.

\section{Clinical Issues and Realities}

Clinically the disorder is missed if not directly assessed; this is seen more often in the developing countries. Patients often do not report the deficits for psychological reasons such as denial, or because of the nature of the disorder and possible anasagnosia. Patients need to be examined on neuropsychological tests in order to be diagnosed as well as defined in the severity of the disorder; tests such as cancellation task, line bisectional task, draw a clock....etc.

Rehabilitation therapists and clinical teams often focus on sensory and motor deficits. It is disturbing that a significant number of patients with neglect are missed or not fully discussed at case conferences, for instance [10], where all interdisciplinary staff are dealing with clinical issues for stroke patients. Furthermore, clinicians are puzzled by the nature of the disorder especially those with less academic or clinical experience. It seems that they have 'avoidant' behaviour towards neglect syndrome. Following evaluation, rehabilitation approaches may be applied.

Rehabilitations techniques are needed to ameliorate the impact of the disorder. This include a number of methods such as; rightwarddeviating prisms (for left neglect), teaching patients to self-alert, repetitive transcranial magnetic stimulation and drug therapy $[4,5]$.

*Corresponding author: Muwafak HAl-Eithan, Consultant Clinical Neuropsychologist Clinical Psychology, Prince Sultan Military Medical City, Riyadh, Saudi Arabia, Tel: +966114777714; Email: al_eithan@yahoo.com

Received March 23, 2016; Accepted May 07, 2016; Published May 10, 2016

Citation: Al-Eithan MH (2016) Facing Neglect after Stroke: Clinical Challenges in Developing Countries. Brain Disord Ther 5: 214. doi:10.4172/2168-975X.1000214

Copyright: $\odot 2016$ Al-Eithan MH. This is an open-access article distributed under the terms of the Creative Commons Attribution License, which permits unrestricted use, distribution, and reproduction in any medium, provided the original author and source are credited. 
Citation: Al-Eithan MH (2016) Facing Neglect after Stroke: Clinical Challenges in Developing Countries. Brain Disord Ther 5: 214. doi:10.4172/2168975X.1000214

Page 2 of 2

Certain rehabilitation approaches have been applied to treat patients with neglect; including; compensatory, substitutive, and restitutive. The first approach aim on using the intact brain functions by using (e.g. visual scanning therapy, optokinetic stimulation, neck muscle vibration, body rotation), by manipulate the sensory input (e.g. visual, tactile/ somatosensory, or the vestibular system) [11]. The second approach relies on methods such as optic devices [11] where improvements have been shown. While the third approach applied methods such as mental imagery [11], with some positive results.

Recent thinking about neglect is that it is that different approaches of treatments may improve some aspects of neglect, but no single therapy approach is widely accepted. Recovery may be spontaneous, but there is evidence indicating that patients continue to have cognitive impairments, mainly relating to attention [12].

\section{Conclusion and Recommendation}

Neglect is a common disorder following stroke. Neglect is the best single predictor of outcome after stroke, and it should be clinically considered in rehab at all treatment stages.

All patients with stroke should be neuropsychologically screened with tests that measure neglect from early stages of care and rehabilitations. Then they should be offered a rehab steps to reduce the impact of the disorder. Patients who have been treated show better outcome and certain improvements last for months and more. Treatment of neglect should be part of all treatments of deficits following stroke.

\section{References}

1. American Heart Association (2014) Heart disease and stroke statistics 2014 update. American Heart Association, Dallas, TX, USA.

2. http://www.emro.who.int/health-topics/stroke-cerebrovascular-accident/index.html

3. Bowen A, McKenna K, Tallis RC (1999) Reasons for variability in the reported rate of the occurrence of unilateral spatial neglect after stroke. Stroke. 30: 1196-1202.

4. Robertson I, Halligan PW (1999) Spatial Neglect, A Clinical Handbook for Diagnosis and Treatment. Psychology Press, UK.

5. Singh-Curry V, Husain M (2008) Rehabilitation of Neglect, Cognitive Neurorehabilitation. Oxford University Press, USA.

6. Karnath HO, Ferber S, Himmelbach M (2001) Spatial awareness is a function of the temporal and not the posterior parietal lobe. Nature 21: 950-953.

7. Buxbaum LI, Ferraro M K, Veramonti T, Farne A, Whyte J, et al. (2004) Hemispatial neglect Subtypes, neuroanatomy, and disability. Neurology 62 749-756.

8. Husain M, Rorden C (2003) Non-spatially lateralized mechanisms in hemispatial neglect. Nat Rev Neurosci 4: 26-36.

9. Vallar G, Perani D (1986) The anatomy of unilateral neglect after righthemisphere stroke lesions. A clinical/CT-scan correlation study in man. Neuropsychologia 24: 609-622.

10. Chen P, McKenna C, Kutlik AM, Frisina PG (2013) Interdisciplinary communication in inpatient rehabilitation facility: evidence of underdocumentation of spatial neglect after stroke. Disabil Rehabil. 35: 1033-1038.

11. Ting DSJ, Pollock A, Dutton GN, Doubal FN, Ting DSW, et al. (2011) Visua neglect following stroke: Current concepts and future focus. Survey of Ophthalmology 56: 114-134.

12. Li K, Malhotra PA (2015) Spatial Neglect. Pract Neurol 1-7. 